\title{
Analysis of differential observations of the cosmological radio background : studying the SZE-21cm
}

\author{
Charles Mpho Takalana*† \\ University of the Witwatersrand \\ E-mail: mtakalana@ska.ac.za \\ Sergio Colafrancesco \\ University of the Witwatersrand \\ E-mail: Sergio.Colafrancesco@its.ac.za

\section{Paolo Marchegiani} \\ University of the Witwatersrand \\ E-mail: Paolo.Marchegiani@wits.ac.za
}

In pursuit of understanding the early Universe and early processes occurring particularly in the Dark Ages (DA) and the Epoch of Reionization (EoR), it is vital that a suitable probe is identified. Probing these epochs will be useful in studies of the origin of first galaxies and most importantly formation of early black-holes. Over the past decade numerous probes have been proposed, with one of the most promising being the SZE$21 \mathrm{~cm}$, a specific form of the Sunyaev-Zeldovich effect (SZE) produced when photons of the $21 \mathrm{~cm}$ background are inverse Compton up-scattered by electrons residing in hot plasma of cosmic structures such as galaxy clusters and active radio galaxies. The $\mathrm{SZE}-21 \mathrm{~cm}$ is calculated in a full relativistic approach of the scattering processes of the CMB photons modified by the cosmological redshifted $21 \mathrm{~cm}$ background in the hot intra-cluster medium of galaxy clusters capturing effects induced by relativistic corrections to this scattering and by multiple scattering effects. We apply image differencing techniques to simulated radio observations of galaxy clusters using the redshifted $21 \mathrm{~cm}$ background, we conduct this making use of the public semi-numeric code 21cmFAST. We are able to achieve subtraction of contaminating foregrounds through pixel by pixel operations on the data retrieved from our simulated data cubes. We demonstrate that SZE-21cm can be recovered through differential observations of the $21 \mathrm{~cm}$ background.

5th Annual Conference on High Energy Astrophysics in Southern Africa

4-6 October, 2017

University of the Witwatersrand (Wits), South Africa

* Speaker.

${ }^{\dagger}$ A footnote may follow. 


\section{Introduction}

Studies of the Dark Ages (DA) and the Epoch of Reionization (EoR) of the Universe will shed light on a large number of fundamental questions in cosmology that will help us understand the properties of the first galaxies and physical mechanisms that give rise to stuctures we observe today. The generally accepted probe for these epochs is the $21 \mathrm{~cm}$ spectral line produced by a spin flip in neutral hydrogen $[24,17,10]$. Low frequency observations of the redshifted $21 \mathrm{~cm}$ line of neutral hydrogen present promising avenues for exploring periods in the Universe that are to date poorly constrained. We have yet to observe the period of formation of the first luminous structures (DA), $20 \lesssim z \lesssim 1100$, and have recently started making ground in exploring the era of the first light that stretches from the formation of these structures to the complete reionization of the intergalactic medium (IGM), $6 \lesssim z \lesssim$ 20. The detectable signal in the frequency range relevant to the DA and EoR is composed of a number of components, namely: instrument response, instrument noise, ionospheric distortions, galactic and extragalactic foregrounds as well as the cosmological signal itself, which is expected to vary with redshift $[3,15]$. Foreground emission is expected to be $4-5$ orders of magnitude stronger than the cosmological signal [20], unscrambling the signal and foregrounds will be non-trivial and foreground subtractions may leave contaminating residuals. Foregrounds will have to be carefully removed with the use of highly accurate and precise cleaning methods as any error could potentially destroy the $21 \mathrm{~cm}$ cosmological signal.

The Sunyaev-Zeldovich Effect (SZE) [21, 22] is a powerful cosmological tool that has been used to identify galaxy clusters; this signal does not vary with distance from the source due to its redshift independent nature. Combining the advantages of the $21 \mathrm{~cm}$ line and the SZE creates a potential probe suitable for studying the Universe at the EoR and DA with the highest precision, we refer to this probe as the SZE-21cm. Coorey 2006 [6] and Colafrancesco et al. 2016 [5] emphasised that differential observations of the SZE-21cm with radio interferometers are less affected by the presence of galactic and extra-galactic foregrounds that are uniform over angular scales larger than a typical cluster and the observations would be less affected by exact calibration of the observed intensity using an external source. Proposed observations of the SZE-21cm can be carried out with interferometers pursued for studying and imaging the $21 \mathrm{~cm}$ line of neutral hydrogen through differential observations toward and away from galaxy clusters (e.g. Low Frequency Array $(\text { LOFAR })^{1}$, Murchison Widefield Array (MWA) ${ }^{2}$, Hydrogen Epoch of Reionization Array $(\text { HERA })^{3}$, Square Kilometre Array $\left.(\mathrm{SKA})^{4}\right)$. Once complete the SKA precusor, HERA, with its 350 elements opperating at frequencies from 50 to $250 \mathrm{MHz}$ will be the first instrument with potential to directly image the EoR [7]. In future SKA-1 Low will cover the $50-350 \mathrm{MHz}$ frequency range, consisting of nearly 130000 antennas across 500 stations with a maximum $65 \mathrm{~km}$ baseline [8], allowing us to image the EoR and DA. The technique

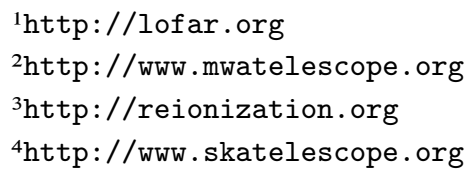


we study here for the SZE-21cm will be applicable to images obtained from both HERA and SKA-1 Low.

This work builds on Colafrancesco et al. 2016 [5] where the theoretical aspect was laid out. We apply the theory here to image differencing of simulated observations of the $21 \mathrm{~cm}$ cosmological signal made using $21 \mathrm{cmFAST}^{5}$. We attempt to recover the SZE- $21 \mathrm{~cm}$ signal from simulated data, and use this to construct expectations for low frequency differential observations. This work links theory, imaging technique and analysis.

This paper is structured as follows. Section 2 unpacks the SZE- $21 \mathrm{~cm}$ signal and formalism for differential observations of the SZE-21cm as well as the $21 \mathrm{~cm}$ cosmological signal itself. Section 3 goes through the methods and results of mock observations of the SZE- $21 \mathrm{~cm}$ and section 4 presents the discussion. We conclude in section 5 . Throughout this paper we assume $\Lambda$ CDM-cosmology with parameters: $h=0.673, \Omega_{m}=0.315, \Omega_{b}=0.0491, \Omega_{\Lambda}=$ $0.685, \sigma_{8}=0.815$ and $n_{s}=0.968$.

\section{Probing the DA and EoR with the SZE-21cm}

\subsection{The $21 \mathrm{~cm}$ Cosmological Signal}

The $21 \mathrm{~cm}$ line is a result of the ground state hyperfine transition of atomic hydrogen, emitting radio waves at $21 \mathrm{~cm}$, a rest frequency $v_{0}=1420.4057 \mathrm{MHz}$. The $21 \mathrm{~cm}$ line allows for mapping of large scale structures including Magellanic clouds, galaxies and galaxy clusters. The $21 \mathrm{~cm}$ signal is expected to be observed as a faint, diffuse background at low frequencies $(\lessgtr 200 \mathrm{MHz})[5]$, its detection will yield information about the global and local properties of the Inter Galactic Medium (IGM). The spin temperature, $T_{s}$, quantifies the excitation temperature for the $21 \mathrm{~cm}$ transition, with $n_{1} / n_{0}=3 \exp \left(-T_{*} / T_{S}\right)$ representing the ratio of the electron density in the triplet state $\left(n_{1}\right)$ to the singlet state $\left(n_{0}\right)$ of the hyperfine level. $T_{*}$ is the temperature corresponding to the $21 \mathrm{~cm}$ wavelength $\left(T_{*}=\frac{h \nu_{0}}{k_{B}}=\right.$ $0.0681 \mathrm{~K}$ ), and $g_{1} / g_{0}=3$ is the ratio of the spin degeneracy factors of the two levels. The spin temperature essentially is a shorthand for the ratio between the occupation number of the two hyperfine levels which establishes the intensity of the radiation emerging from a cloud of neutral hydrogen [26] and is determined by three processes: absorption of CMB photons; collisions between the particles, which is defined by the gas kinetic temperature, $T_{k}$; and scattering of ambient UV (Ly- $\alpha$ ) photons, $T_{\alpha}$. Assuming $T_{\alpha}=T_{k}$ (Field et al. 1958 [11]):

$$
T_{S}=\frac{T_{C M B}+y_{k} T_{k}+y_{\alpha} T_{k}}{1+y_{\alpha}+y_{k}}
$$

$y_{\alpha}$ and $y_{k}$ are the Ly- $\alpha$ and kinetic coupling terms respectively. The Ly- $\alpha$ coupling term $y_{\alpha}$ is due to the Wouthuysen-Field process (Ly $\alpha$ pumping) occurring through collisions and scattering of $\operatorname{Ly} \alpha$ photons coupling the spin temperature to the gas temperature, $T_{k}$, making neutral hydrogen visible in absorption or emission as a result of the gas being

${ }^{5}$ homepage.sns.it/mesinger/ 
colder or hotter than that of the CMB $[9,11,13,25]$. Changes in the CMB temperature, the kinetic temperature, and spin temperature $\left(T_{s}\right)$ lead to interesting physical features of the HI $21 \mathrm{~cm}$ signal. The quantity that $21 \mathrm{~cm}$ interferometers will measure is the $21 \mathrm{~cm}$ differential brightness temperature $\delta T_{b} \equiv T_{b}-T_{C M B}$, a deviation from the CMB temperature also known as the global brightness temperature [26]

$$
\delta T_{b} \approx 25 x_{H I}(1+\delta)\left(\frac{1+z}{10}\right)^{1 / 2}\left[1-\frac{T_{C M B}}{T_{S}}\right]\left[\frac{H(z) /(1+z)}{d v_{\|} / d r_{\|}}\right] m K .
$$

Equation 2.2 is a mixture of cosmology and astrophysics dependent terms. $\delta T_{b}$ is controlled by different contributions at different stages of its evolution. At high redshifts $(z \gtrsim 10)$ the neutral fraction $\left(x_{H I}\right)$ is approximately one, $\delta T_{b}$ is proportional to the density fluctuations, and this measurements places it as an excellent cosmological probe. At low redshifts $(\mathrm{z} \lesssim 10)$ the Universe is ionized and measurements of $\delta T_{b}$ are dominated by the contrast between the neutral and ionized regions, and this probes the astrophysical source of ionization. Equation 2.2 tells us that the $21 \mathrm{~cm}$ radiation can only be observed if it has a temperature that differs from that of the CMB, no signal is expected when $T_{S} \sim T_{C M B}[11,12,25]$.

Semi-numeric modeling tools such as 21cmFAST [16] and Simfast21 [18, 19] simulate the observable $21 \mathrm{~cm}$ differential brightness temperature $\delta T_{b}$ which is detected differentially as a deviation from the CMB temperature. The $21 \mathrm{~cm}$ global brightness temperature signal we expect to observe is in figure 1 , this was obtained from our simulation run of $21 \mathrm{cmFAST}$ which we use as the basis of this text, an example of a simulated $30 \mathrm{Mpc}$ data cube at 114.27 MHz is presented along side the spectrum, figure 1.

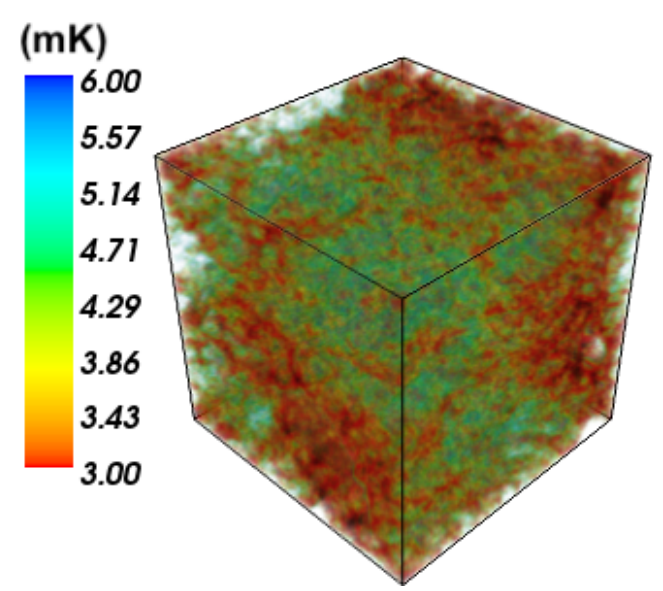

(a)

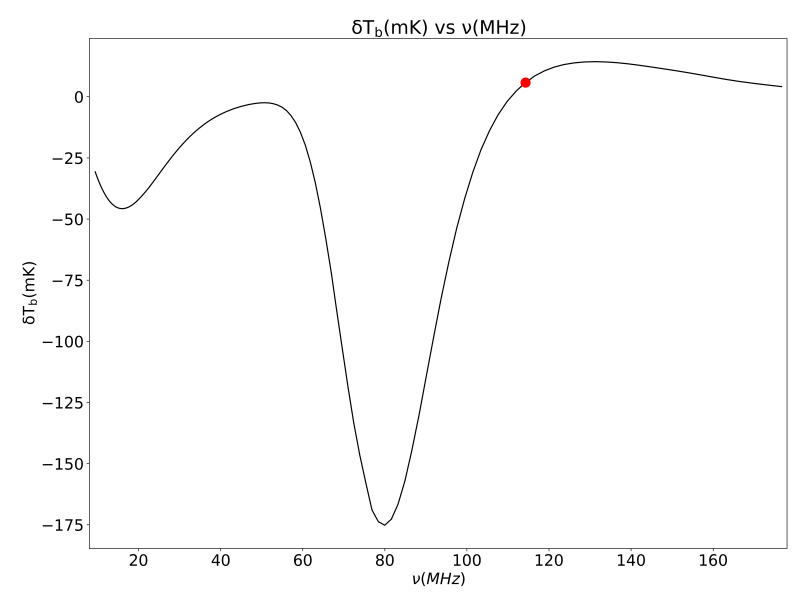

(b)

Figure 1: (a) Simulated 21cm Brightness temperature 3D volume at 114.27 MHz. (b) The sky averaged $21 \mathrm{~cm}$ brightness temperature offset from the CMB spectrum as a function of frequency from our simulations. 


\subsection{The SZE-21cm}

Inverse Compton scattering slightly alters the incident Planck spectrum, changing the apparent brightness of the Cosmic Microwave Background (CMB) radiation towards a reservoir of hot plasma [2] imprinting on it a unique spectral signature known as the Sunyaev Zeldovich effect (SZE) [21, 22, 23, 27]. Initially proposed by Cooray 2006 [6] and later redefined taking a relativistic approach by Colafrancesco et al. 2016 [5], the SZE-21cm is an alternative method that can be used to overcome foreground challenges faced when studying the early epochs using the $21 \mathrm{~cm}$ line because it is a differential measure of the radio background spectrum on and off an area of the sky containing the cosmic structure of interest. The SZE-21cm is the spectral distortion of the CMB spectrum altered by physical effects occurring during the epoch related to the emergence of the $21 \mathrm{~cm}$ radiation background, photons of the CMB radiation are scattered by electrons in the hot intracluster gas. Differential observations of this signal with low frequency radio interferometers are less affected by large-scale foregrounds and exact calibration of the observed intensity $[5,6]$. In this section we go through the derivation for the SZE- $21 \mathrm{~cm}$, the reader is refered to Colafrancesco et al. 2003 and 2016 [5, 4] for full derivations in the relativistic limit. The scattering optical depth of a cluster which contains gas integrated along the line of sight of the electron concentration $n_{e}(\boldsymbol{r})$ is given by

$$
\tau_{e}=\int n_{e}(r) \sigma_{T} d l
$$

where $\sigma_{T}$ is the Thomson cross section; this is required to calculate the electron redistribution function $P(s)$ as the Poissonian probability containing relativistic corrections essential for correctly describing the Compton scattering produced by relativistic electrons in hot plasma

$$
P(s)=\sum_{n=0}^{+\infty} \frac{e^{-\tau} \tau^{n}}{n !} P_{n}(s)
$$

where $P_{n}(s)$ is the probability function to have $n$ scatterings that is given by the convolution of $n$ times the single scattering probability $P_{1}(s)$ (e.g. Colafrancesco et al. 2003 [4]), and the inverse Compton scattering process yields the probability of a logarithmic shift $s=\ln \left(\frac{v^{\prime}}{v}\right)$ in the photon frequency. The general form of the spectral distortion of the CMB due to the SZE is given by

$$
I(x)=\int_{-\infty}^{+\infty} I_{0}\left(x e^{-s}\right) P(s) d s
$$

where the normalised frequency is represented as $x=\frac{h v}{k T_{C M B}}$ and $I_{0}$ is the specific intensity of the incident CMB radiation field [5, 4]. The general form of the SZE is than given by the difference: 


$$
\Delta I(x)=I(x)-I_{0}(x)
$$

For the standard case the incoming spectrum is the standard CMB spectrum:

$$
I_{0, s t}(x)=2 \frac{\left(K T_{0}\right)^{3}}{(h c)^{2}} \frac{x^{3}}{e^{x}-1}:
$$

putting this into equations 2.5 and 2.6 we get the standard SZE, $\Delta I_{s t}(x)$. Calculations that take into consideration modifications to the CMB spectrum by neutral hydrogen can be made to establish the low frequency spectrum emerging from a galaxy cluster post scattering. The expected modification to the input spectrum is a few milli-Kelvin correction to the temperature of the CMB black body spectrum. Coorey 2006 [6] gave a description of the SZE-21cm in a non-relativistic approximation neglecting effects induced by relativistic corrections, these are instead accounted for by Colafrancesco et al. 2016 [5]. The CMB spectrum modified by neutral hydrogen during the DA and EoR is given by

$$
I_{0, \bmod }(v)=I_{0, s t}(v)+\delta I(v)
$$

where $\delta I(v)$ can be written in terms of the brightness temperature change relative to the CMB

$$
\delta T_{b}(v)=\frac{c^{2}}{2 k v^{2}} \delta I(v)
$$

With $I_{0, \bmod }(v)$ as our incoming radiation spectrum and the SZE- $21 \mathrm{~cm}$ reads

$$
\Delta I_{\text {mod }}(v)=I_{\text {mod }}(v)-I_{0, \bmod }(v) .
$$

Applying equation 2.9, we can rewrite equation 2.10 in terms of temperature as

$$
\Delta T_{\text {mod }}(v)=T_{\text {mod }}(v)-T_{0, \bmod }(v) .
$$

\section{Differential Analysis techniques for the SZE-21cm}

This work makes use of 21cmFAST [16] which employs semi-numerical approaches to produce large-scale simulations making use of: analytical prescriptions, the excursion set formalism (used to predict the clustering properties of dark matter halos relative to the dark matter), and perturbation theory to produce evolved 3D realizations of spin temperature fields, density, peculiar velocity and ionization, which when combined can be used in the computation of the $21 \mathrm{~cm}$ brightness temperature described by equation 2.2 . These simulations produce $\delta T_{b}$ cubes (signal as a function of frequency). We produced three dimensional 
$30 h^{-1}$ comoving Mpc simulation cubes, from redshift $\mathrm{z}=6.89$ up to redshift $\mathrm{z}=149.74$, these all make up our mock observations. The initial grid of the cubes is $460^{3}$ voxels which are smoothed down to a $115^{3}$ grid. The standard CMB required for analysis of the SZE is not taken into account by $21 \mathrm{cmFAST}$ and therefore we add the standard CMB to the $21 \mathrm{~cm}$ brightness temperature in all the simulated cubes through equation 2.8; the result of this are CMB cubes modified by neutral hydrogen during the DA and EoR.

\subsection{Temperature variations in $21 \mathrm{~cm}$ simulated observations}

Temperature variations in the $\delta T_{b}$ cubes were of order $10^{-1}-10^{-3}$, the order of these variations create a great concern as they lead to incorrect values for the SZE- $21 \mathrm{~cm}$ when conducting our differential observations. This arises from the SZE-21cm being a CMB effect and that the CMB primodial fluctuations are of order $10^{-5}$, hence the background we substract through the differencing procedure need to contain similar order variations to the CMB. The source of variations in these cubes are significant degeneracies that exist between parameters that govern the mean brightness spectrum leading to inhomogeneities which can be observed by interferometers pursued for $21 \mathrm{~cm}$ observations [14]. The inhomogeneities are induced by fluctuations from various regions of the IGM which include contributions from different physical properties including velocity gradients, temperature (gas \& spin), ionization state and density. Establishing signatures associated with the mean brightness spectrum independent of spacial variations could play a role in breaking some of the degeneracies. Inhomogeneities generate anisotropies in the brightness temperature during the DA and EoR [14], in addition to this there are fluctuations due to gravitational lensing through scattering in galaxies. We do not however focus on how the degeneracies can be broken as this is beyond the scope of this text. We employ pixel replacements which not only take care of variations due to inhomogeneity but also bright sources and foregrounds. In the context of this work bright sources in the background image were identified as foreground contaminants, and hence were removed from the maps to prevent them from contributing to the brightness temperature fluctuations. Taking a cube slice away from the inserted cluster as the background image: pixels $T_{0, \text { mod }_{i}}$ in the background image were replaced with the mean cube temperature $\bar{T}_{0, \text { mod }}$ as described below:

$$
\text { if } \begin{aligned}
\frac{T_{0, \text { mod }_{i}}-T_{c m b}}{T_{c m b}} & \gtrsim 10^{-5} \text { then } \\
T_{0, \text { mod }_{i}} & =\bar{T}_{0, \text { mod }}
\end{aligned}
$$

this was done to ensure that the background remains with variations of the same order as the CMB. Figure 2 shows a background image before and after the variations are dealt with.

\subsection{Method for simulated differential observations for the SZE-21cm}

Our differential observing technique hinges on equation $(2.11), T_{m o d}(v)$ represents the patch of sky that contains a galaxy cluster, and $T_{0, \bmod }(v)$ represents a patch of sky containing only the background. The cluster signal we insert into our cube was calculated using the mean $21 \mathrm{~cm}$ brightness temperature of each cube through the spectrum in figure 1 , this 


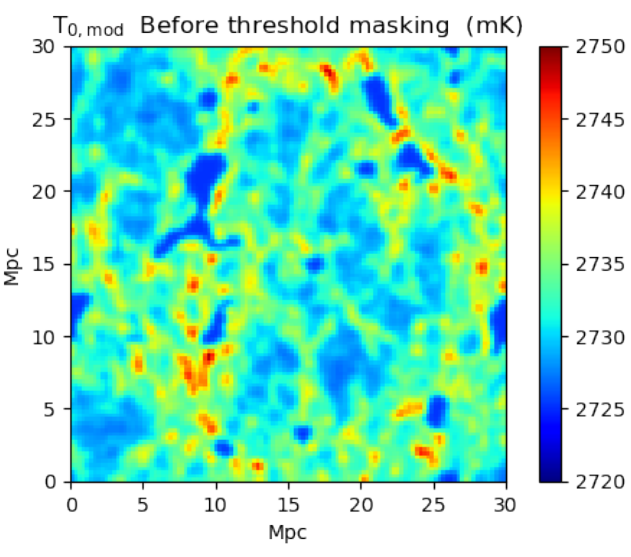

(a)

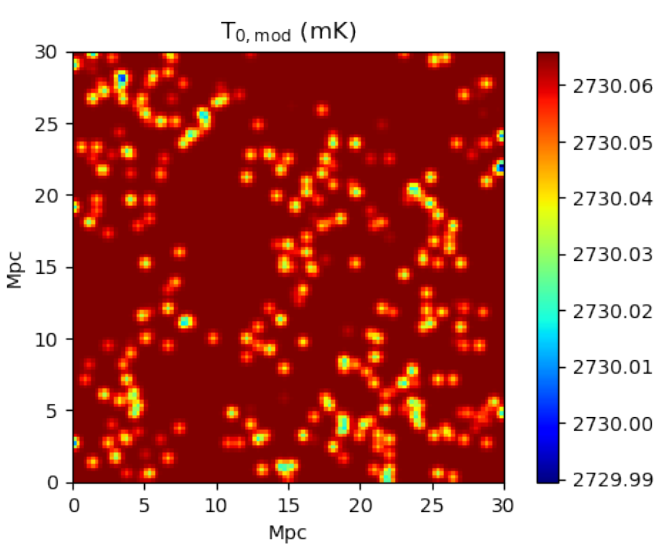

(b)

Figure 2: a: Original 21 cm background image from an area in the simulated cube before pixel replacement with temperature variations of order $10^{-1}-10^{-3}$ at $114.27 \mathrm{MHz}$, b: The $21 \mathrm{~cm}$ background image after pixels were replaced such that the cube variations were of order $10^{-5}$ at $114.27 \mathrm{MHz}$.

cluster signal is $T_{\bmod }(v)$ in equation 2.11. The inserted spherical cluster in figure 3 (a) has a temperature of $10 \mathrm{keV}$, optical depth of $10^{-3}$ and a radius of $3 \mathrm{Mpc}$. These values are typical of rich clusters where the SZE has been observed; the SZE we consider at the cluster is due only to the thermal effect. The slice away from the cluster represents our background in figure 2 (b) with the temperature variations taken care off. Following the subtraction of the background image from the cluster image through equation 2.11 we are left with the SZE-21cm signal at the cluster and residual foregrounds, the result image of this subtraction is figure $3(\mathrm{~b})$.

The differencing procedure was followed for cubes of frequency range 6 to $180 \mathrm{MHz}$, the SZE-21cm was measured in each cube and used to construct the spectrum in figure 4 which we discuss in section 4 .

\section{Discussion on SZE-21cm simulation results}

The SZE-21cm can be used to study in depth the physical history of the EoR and DA. The SZE-21cm was obtained by measuring the signal after image differencing in section 3, we now discuss how this signal can be used to directly establish the global $21 \mathrm{~cm}$ spectrum, and its dependencies on the properties of the plasma in galaxy clusters. Figure 4 shows us the SZE-21cm spectrum obtained by following the differencing procedure in the previous section from 6 to $180 \mathrm{MHz}$.

The observables in the spectrum are listed below:

- The SZE-21cm measured at the cluster (black dots in figure 4) agrees closely with the calculations done in Colafrancesco et al. 2016 [5], represented by the green line. 


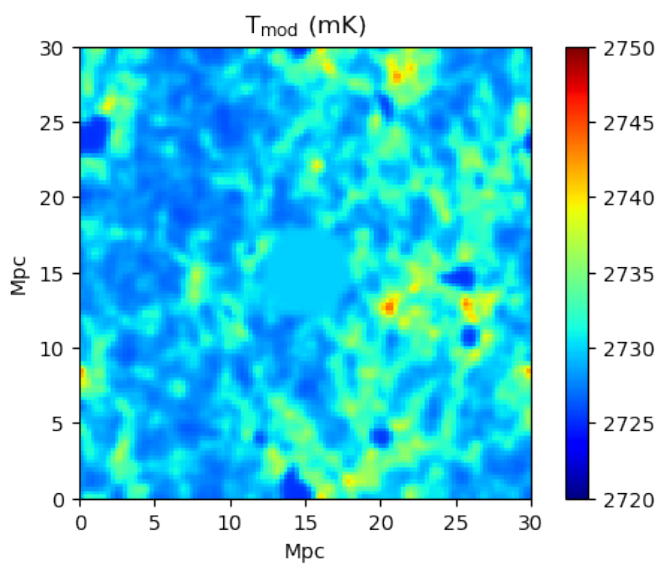

(a)

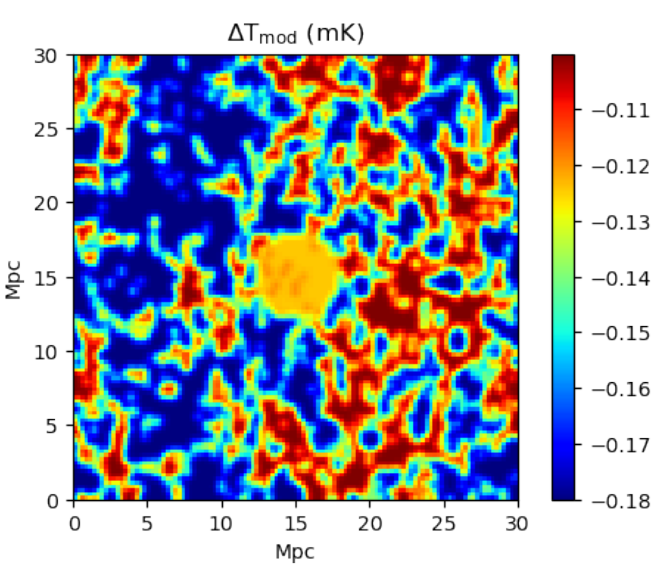

(b)

Figure 3: In (a) we have an image of the inserted cluster in the presence of a $21 \mathrm{~cm}$ background at 114.27, and in (b) we have the final image of the cluster SZE-21cm obtained from the image differencing procedure at $114.27 \mathrm{MHz}$.

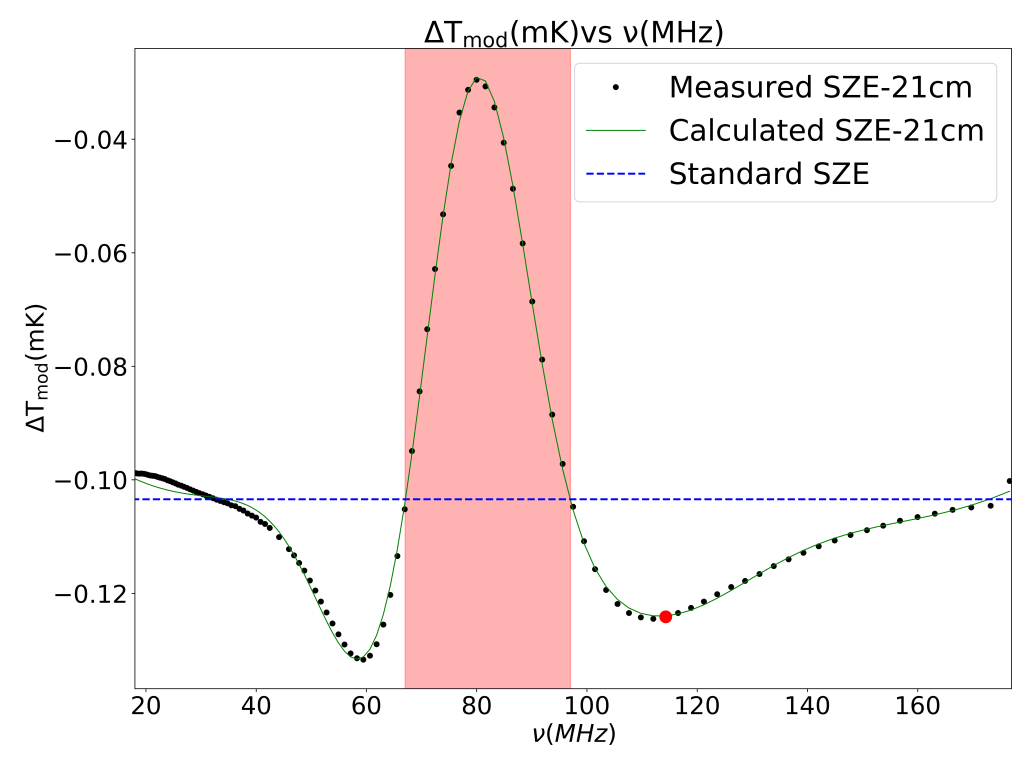

Figure 4: The SZE-21cm measured following the image differencing technique for a cluster of $10 \mathrm{keV}$, an optical depth of $10^{-3}$. The red area represents where the SZE-21cm is bigger than the standard SZE. The green line is the best-fit line of theoretically calculated data points (Colafrancesco et al. 2016 [5]), the black dots are points measured from the images and the blue dashed line is the standard SZE. The red point corresponds to the sample figure at $114.27 \mathrm{MHz}$. 
This is expected as the formalism used in the calculations is the same as that used for the simulations.

- The SZE-21cm is bigger than the standard SZE at $67 \lesssim v \lesssim 97 \mathrm{MHz}$. The standard SZE is bigger than the SZE- $21 \mathrm{~cm}$ at $v \lesssim 67$ and $97 \lesssim v \lesssim 180$.

Something that immediately stands out about the SZE-21cm spectrum is its resemblance of an inverted $21 \mathrm{~cm}$ brightness temperature input spectrum, figure 1. It is in this feature that we are able to use the SZE- $21 \mathrm{~cm}$ spectrum to narrate evolution of the $21 \mathrm{~cm}$ signal itself. At frequencies where the curvature of the input $21 \mathrm{~cm}$ brightness temperature spectrum is negative, a smaller number of photons are present at neighbor frequencies with respect to the standard CMB spectrum. For frequencies $30 \lesssim v \lesssim 67 \mathrm{MHz}$ and $97 \lesssim v \lesssim 174 \mathrm{MHz}$, the input spectrum has negative curvature, here the SZE-21cm is smaller than the standard SZE, where else for frequencies $67 \lesssim v \lesssim 97 \mathrm{MHz}$ the input spectrum has positive curvature and the SZE-21cm is bigger than the standard SZE. Through the subtraction procedure guided by equation 2.11 we find that the minimum point of the input spectrum, figure 1 , corresponds to the maximum point of the SZE- $21 \mathrm{~cm}$ spectrum, figure 4 . Where a smaller number of photons exist with respect to the standard CMB, the resulting emission of the SZE-21cm is bigger than the standard SZE. Where the input spectrum is maximum, the SZE-21cm spectrum is at its minimum, a larger quantity of photons exist with respect to the standard SZE. The result difference images show some features associated with the subtracted background, these features are dominantly seen inside and outside the cluster region where the standard SZE is bigger.

\subsection{Detectability of the signal with SKA telescopes}

The SZE-21cm and standard SZE are detectable with the SKA instrument; figure 5(a) shows detectability of the non-modified SZE spectrum, $\Delta I_{s t}$, and modified SZE spectrum, $\Delta I_{\text {mod }}$, compared with the sensitivities of SKA telescopes, SKA-50\%, SKA1-Low, and SKA2. We use the SKA1 System Baseline document to extract the instrument performances [8]. Observing at $100 \mathrm{kHz}$ bandwidth, with 1000 hours integration time, 2 polarizations, no taper, no weight: the SZE-21cm for a $10 \mathrm{keV}$ cluster will be detectable with SKA-50\% at frequencies $\gtrsim 90 \mathrm{MHz}$, SKA1-Low at frequencies $\gtrsim 85 \mathrm{MHz}$, and SKA2 will be able to detect the signal below $60 \mathrm{MHz}$ in the DA. Figure $5(\mathrm{~b})$ gives us an idea of the frequency bands where we will be able to distinguish between the SZE-21cm and the standard effect, that is the detectable difference. The SZE- $21 \mathrm{~cm}$ is weaker compared to the standard SZE due to the Wouthuysen [25] effect at around $80 \mathrm{MHz}$, this is a good frequency to detect the difference between the two signals; another range is at $110-120 \mathrm{MHz}$, where the SZE-21cm appears stronger due to ionization effects. With SKA1-low sensitivity at its high frequency band, the best frequency range to study the SZE-21cm is $\gtrsim 100 \mathrm{MHz}$. With SKA2 however we expect improved results, the difference between the SZE-21cm and the standard effect will be detectable at $v \gtrsim 75 \mathrm{MHz}$. 


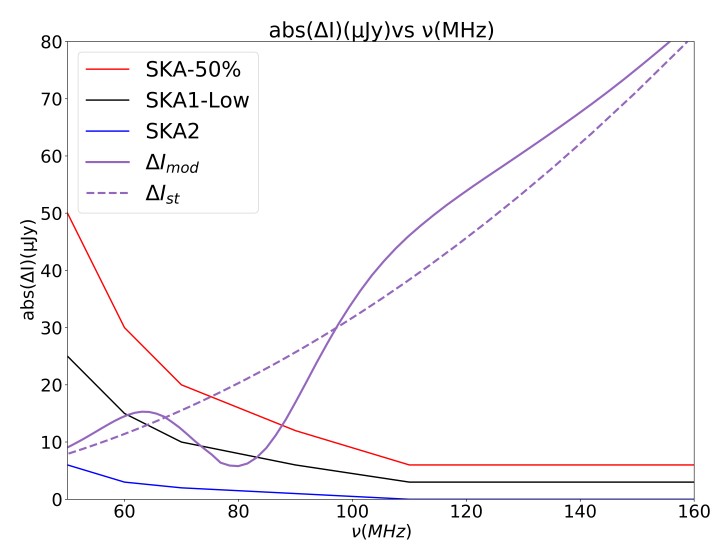

(a)

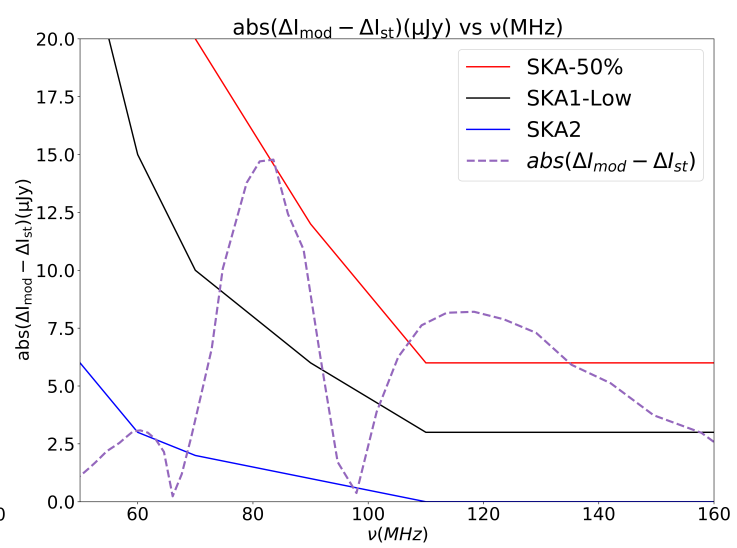

(b)

Figure 5: (a) Sensitivity of SKA telescopes to the SZE-21cm and the standard SZE, the dashed purple line is the Standard SZE, the solid purple line is the $S Z E-21 \mathrm{~cm}$. (b) Sensitivity of SKA telescopes to difference of the SZE-21cm and standard SZE, the dashed purple line is the absolute difference between the SZE-21cm and the standard SZE. In both figures the red, black and blue lines represent the telescope sensitivity as shown in the figure legend.

\section{Conclusion}

The application of the differential technique to simulated $21 \mathrm{~cm}$ observations shows that the SZE-21cm can be detected and measured directly from observation and may therefore be used to construct the global $21 \mathrm{~cm}$ cosmological signal. The technique can further be bettered by averaging over a larger number of clusters which will be useful in dealing with the statistics of the SZE-21cm.

\section{Acknowledgements}

This work is based on the research supported by the South African Research Chairs Initiative of the Department of Science and Technology and National Research Foundation of South Africa (Grant No 77948). C.M., S.C. \& P.M. acknowledge support from the Department of Science and Technology/National Research Foundation (DST/NRF) Square Kilometre Array (SKA) post-graduate bursary initiative under the same Grant.

\section{References}

[1] Aghanim, N., Majumdar, S., and Silk, J. (2008). Reports on Progress in Physics, 71. 066902.

[2] Birkinshaw, M., (1999). Physics Reports, 310. 97-195.

[3] Ciardi, B., and Madau, P., (2003). ApJ, 596. 1-8.

[4] Colafrancesco, S., Marchegiani, P., and Palladino, E. (2002). Astron.Astrophys., 397 27-52

[5] Colafrancesco, S., Marchegiani, P., and Emritte, M. (2016). A\&A, 595 A21. 
[6] Cooray, R.A. (2006). Phys.Rev., 470:103001. 1-6.

[7] DeBoer D.R., Parsons A.R., AguirreJ.E., Alexander P., Ali Z.S., and others (2017). The Astronomical Society of the Pacific, 129:045001. 27.

[8] Dewdney P., Turner W., Millenaar R., McCool R., Lazio J., and Cornwell T.(2012). Phys.Rev., PoS(AASKA14). 170.

[9] Evoli, C. (2014). JCAP, 11. 024

[10] Ewen, H.I., and Purcell, E.M., (1951). Nature, 168. 356.

[11] Field, G. (1958). ApJ, 129. 536.

[12] Furlanetto, S., Oh, S., and Briggs, F. (2006). Phys.Rept., 433. 181-301.

[13] Hirata, C. (2006). Mon. Not. R. Astron. Soc., 367. 259-274.

[14] McQuinn M., Zahn O., Zaldarriaga M., Hernquist L., Furlanetto S.R. (2005). Astrophys.J., 653. $815-830$.

[15] Mellema, G., Ilian, T.I., Pen, U., and Shapiro, P.R., (2006). Mon. Not. R. Astron. Soc., 000. 1.

[16] Mesinger, A., Furlanetto, S.R., and Cen, R., (2010). Mon. Not. R. Astron. Soc., 411. 955-972.

[17] Muller, C.A., and Oort, J.H. (1957). Bulletin of the Astronomical Institutes of the Netherlands, 13. 151-195.

[18] Santos, M., Amblard, A., Pritchard, J., Trac, H., Cen, R., and Cooray, A. (2008). AJ.

[19] Santos, M., Ferramacho, L., Silva, M., Amblard, A., and Cooray, A. (2010). MNRAS page

[20] Shaver, P. A., Windhorst, R. A., Madau, P., and de Bruyn, A. G., (1999). A\&A, 345. 380.

[21] Sunyaev, M.A. and Zel'dovich, Y. (1980). Ann. Rev. Astron. Astrophys, 18. 537-560.

[22] Sunyaev, M.A. and Zel'dovich, Y. (1970). Comments Astrophys. Space Phys., 2. 66-74.

[23] Sunyaev, M.A. and Zel'dovich, Y. (1972). Comments Astrophys. Space Phys., 4. 173-78.

[24] van de Hulst, H. C., (1945). Ned.Tijd.Natuurkunde, vol.11. 210.

[25] Wouthuysen, S. (1952). ApJ, 57. 31-32.

[26] Zaroubi, S. (2013). The first Galaxies, Astrophysics and Space Science Library, 396. 45-96.

[27] Zel'dovivch, Y. and Sunyaev, R. (1969). Astrophys. Space Sci., 4. 301. 